\title{
Short Duration Gamma-Ray Bursts and Their Outflows in Light of GW170817
}

\author{
Davide Lazzati * \\ Department of Physics, Oregon State University, Corvallis, OR, United States
}

\section{OPEN ACCESS}

Edited by:

Bruno Giacomazzo,

University of Milano-Bicocca, Italy

Reviewed by:

Maria Dainotti,

Jagiellonian University, Poland

Herman J. Mosquera Cuesta,

Departamento Administrativo

Nacional de Ciencia, Tecnologia e Innovacion, Colciencias, Colombia

*Correspondence:

Davide Lazzati

davide.lazzati@oregonstate.edu

Specialty section:

This article was submitted to

Cosmology,

a section of the journal

Frontiers in Astronomy and Space

Sciences

Received: 01 July 2020

Accepted: 11 September 2020

Published: 09 November 2020

Citation:

Lazzati D (2020) Short Duration

Gamma-Ray Bursts and Their

Outflows in Light of GW170817.

Front. Astron. Space Sci. 7:578849.

doi: 10.3389/fspas.2020.578849
The detection of GW170817, it's extensive multi-wavelength follow-up campaign, and the large amount of theoretical development and interpretation that followed, have resulted in a significant step forward in the understanding of the binary neutron star merger phenomenon as a whole. One of its aspects is seeing the merger as a progenitor of short gamma-ray bursts (SGRB), which will be the subject of this review. On the one hand, GW170817 observations have confirmed some theoretical expectations, exemplified by the confirmation that binary neutron star mergers are the progenitors of SGRBs. In addition, the multimessenger nature of GW170817 has allowed for gathering of unprecedented data, such as the trigger time of the merger, the delay with which the gamma-ray photons were detected, and the brightening afterglow of an off-axis event. All together, the incomparable richness of the data from GW170817 has allowed us to paint a fairly detailed picture of at least one SGRB. I will detail what we learned, what new questions have arisen, and the perspectives for answering them when a sample of GW170817-comparable events have been studied.

Keywords: gamma-ray bursts, relativistic astrophysics, hydrodynamics, gravitational waves, binary mergers, transient sources

\section{INTRODUCTION}

Gamma-ray bursts (GRBs) are some of the most energetic explosions in the present day Universe, characterized by the release of large amounts of energy, within a few milliseconds to tens of seconds, resulting in the acceleration of relativistic outflows and the release of high-energy photons (Fishman and Meegan, 1995; Piran, 1999; Mészáros, 2002; Gehrels et al., 2009; Kumar and Zhang, 2015). They can be divided in at least two classes, based on the duration of their prompt phase, in which their emission is concentrated in the hard X-ray and gamma-ray bands and is characterized by fast variability (Kouveliotou et al., 1993). Long duration GRBs last $2 \mathrm{~s}$ or more, while short duration GRBs (SGRBs) last between a few milliseconds and $2 \mathrm{~s}$. Alternative classifications have also been introduced, considering, e.g., short GRBs with extended emission (Norris and Bonnell, 2006; Dainotti et al., 2010; Norris et al., 2010; Barkov and Pozanenko, 2011; Dainotti et al., 2017), or attempting a more physical classification based on inferred progenitor properties (Lü et al., 2010; Bromberg et al., 2013).

In the last two and a half decades, the study of GRBs has concentrated on long duration GRBs, and a general consensus has grown around a model in which these events are associated with the collapse of the core of massive stars (Woosley and Bloom, 2006). While the collapse of most massive stars would ignite a core-collapse supernova, those that are fastly spinning and metal poor could also trigger a long duration GRB, powered by a compact central engine (Woosley, 1993; 

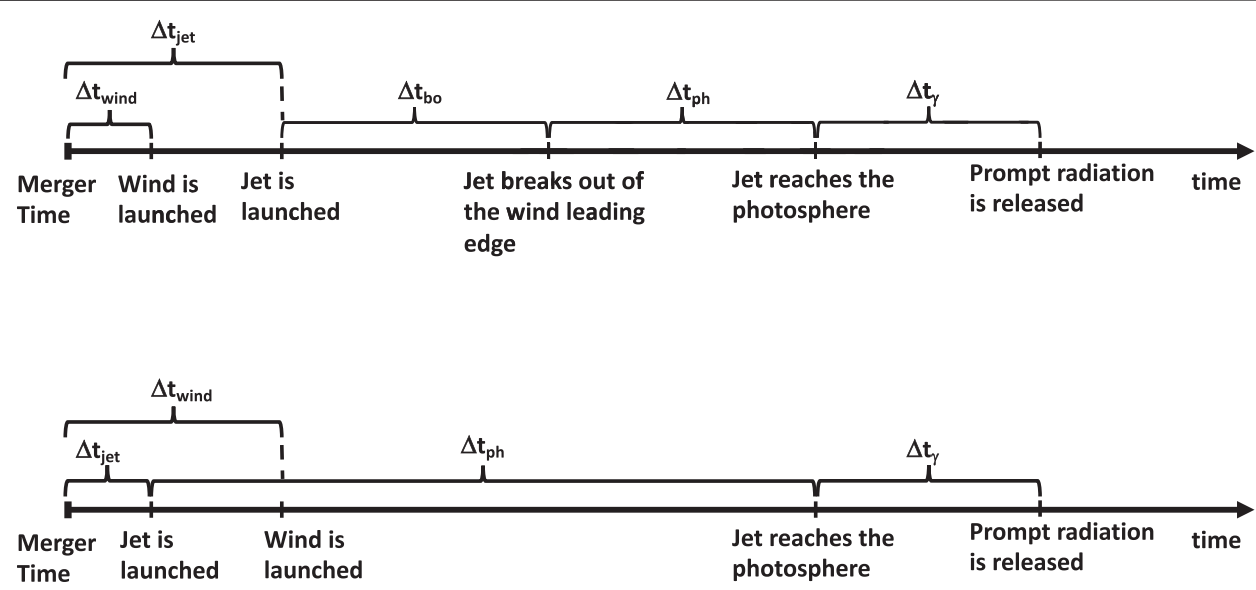

FIGURE 1 | The two possible timelines with all the phases that may contribute to the detected delay $\Delta t_{\mathrm{GW}-\gamma}$. Due to the presence of a structured outflow, GW170817 most likely followed the top timeline. The relative contribution of the various phases is a matter of debate, but consensus is growing around $\Delta t_{\text {wind }}<\Delta t_{\text {jet }} \ll 1$ $\mathrm{s}, \Delta t_{\mathrm{bo}} \ll 1 \mathrm{~s}, \Delta t_{\gamma} \sim 0$, and $\Delta t_{\mathrm{ph}} \sim \Delta t_{\mathrm{GW}-\Gamma}$. The meaning of all the symbols is explained in Section 2

Woosley and Heger, 2006; Yoon et al., 2006). Whether the central engine is a fastly spinning, highly magnetized neutron star (NS) (Bucciantini et al., 2008; Bucciantini et al., 2009; Metzger et al., 2011) or an accreting black hole (BH) (Woosley, 1993; Lee et al., 2000; Lei et al., 2013) is the matter of open debate.

The interest on SGRBs had increased in the last decade, initially as a consequence of the launch of the Fermi satellite, which had a higher efficiency for detecting and localizing them compared to its predecessors (Meegan et al., 2009). More recently, the theoretical expectation that SGRBs had to be associated with the merger of binary NS systems (or, perhaps, system made by a BH and a NS) (Belczynski et al., 2006; Lee and Ramirez-Ruiz, 2007; Fong and Berger, 2013; Giacomazzo et al., 2013; Ruiz et al., 2016; Ciolfi, 2018) has made them the expected and highly anticipated high-frequency counterparts of gravitational wave sources (Nakar et al., 2006; Kiuchi et al., 2010; Schutz, 2011). Such expectations were supported by energetic and temporal arguments. Powering a GRB requires a large amount of energy, comparable to the rest mass of a stellar object converted to energy. In addition, said energy needs to be released in a matter of a fraction of a second, at least for SGRBs. Naked compact objects (NS and BH) are the only available candidates that can offer the required energy within a region of less than a light second. However, isolated $\mathrm{NS}$ and $\mathrm{BH}$ are unlikely progenitors, since some catastrophic event needs to take place to cause the sudden release of a large fraction of their total energy. Binary mergers are therefore a natural candidate, when at least one of the two members is a NS, since a binary $\mathrm{BH}$ system would merge in a bigger $\mathrm{BH}$ that would swallow all the matter and energy, instead of ejecting them as a relativistic outflow. ${ }^{1}$

All these expectations were confirmed by the detection of GW170817 (Abbott et al., 2017a) and its associated GRB

${ }^{1}$ Some have suggested, however, that even binary $\mathrm{BH}$ mergers could produce a weak electromagnetic transient, under certain conditions (Perna et al., 2016; Liu et al. 2016; Zhang, 2016).
GRB170817A (Abbott et al., 2017b; Goldstein et al., 2017; Savchenko et al., 2017; Zhang et al., 2018), afterglow, and kilonova (KN) (Abbott et al., 2017c; Abdalla et al., 2017; Alexander et al., 2017; Arcavi et al., 2017; Coulter et al., 2017; Cowperthwaite et al., 2017; Evans et al., 2017; Haggard et al., 2017; Hallinan et al., 2017; Kasen et al., 2017; Kim et al., 2017; Margutti et al., 2017; Pian et al., 2017; Siebert et al., 2017; Smartt et al., 2017; Soares-Santos et al., 2017; Tanvir et al., 2017; Troja et al., 2017; Valenti et al., 2017; D'Avanzo et al., 2018; Margutti et al., 2018; Mooley et al., 2018a; Mooley et al., 2018c; Lyman et al., 2018; Resmi et al., 2018; Villar et al., 2018; Ghirlanda et al., 2019; Lamb et al., 2019a). In this contribution I will review the key observations of GW170817 as a SGRB (also known as GRB170817A), the questions that were answered, and the new ones that were spurred, and briefly discuss what more insight is expected from the detection of more systems akin to GW170817 in future $\mathrm{GW}$ observing runs.

\section{BEFORE THE PROMPT EMISSION}

In this Section 1 will review the physics of the SGRB outflow before the prompt emission phase begins, as it happened in GW170817. First of all, there is little doubt that the GW signal of GW170817 came from a binary compact merger, and that the masses of the two compact objects are compatible with being NS (Abbott et al., 2017a; Abbott et al., 2017b; Abbott et al., 2017c). The GW signal by itself does not allow to distinguish between NSs and BHs, but the richness of the electromagnetic signal that followed requires the presence of baryonic matter, and therefore at least one of the two components of the binary had to be a NS. Most likely they were both NSs (Coughlin and Dietrich, 2019).

\subsection{The Time Delay}

Besides the identification of the progenitor, a very important piece of information that GW170817 provided is the merger time, 
which allowed for the measuring of the time delay between the GWs and the gamma-ray signals. This delay, which we indicate as $\Delta t_{\mathrm{GW}-\gamma}$ can be due to several reasons, as detailed below and shown in Figure 1 (Granot et al., 2017; Lin et al., 2018; Zhang, 2019; Lazzati et al., 2020; Lucca and Sagunski 2020).

- Engine Delay-While the time of the merger is the earliest time at which the jet from the central engine can be produced, there is the possibility of some delay (Cook et al., 1994; Lasota et al., 1996; Vietri and Stella, 1998; Ciolfi and Siegel, 2015). Such delay is difficult to predict theoretically but can be likely due either to the need of a transition in the engine itself or to the need of amplifying the magnetic field to a value large enough to launch a jet. The former can be quite long, up to years, and usually invokes a metastable, fastly spinning NS that collapses into a $\mathrm{BH}$ when its rotation period is increased by either internal or external torques. We indicate this delay time as $\Delta t_{\text {eng }}$.

- Wind Delay-Owing to the detection of a KN and an offaxis SGRB from a structured outflow, we know that GW170817 ejected a non-relativistic wind. There can be a delay in launching such a wind as well, and we indicate it as $\Delta t_{\text {wind }}$. It should be noted, however, that this delay can in principle be negative since the NS surfaces are tidally shredded in the last few orbits before the merger.

- Breakout delay-If the wind is ejected before the jet, then the jet has to propagate through the wind. The propagation happens at sub-relativistic speed, causing a delay of the head of the jet with respect to the GW signal that travels at the speed of light (Matzner, 2003; Morsony et al., 2007; Bromberg et al., 2011; Lazzati and Perna, 2019). We indicate the time it takes for the jet head to cross the wind as $\Delta t_{\mathrm{bo}}$. The jet-wind interaction also causes the development of a cocoon (Ramirez-Ruiz et al., 2002), confined by the surrounding wind. This leads to the development of a structured outflow that maintains a bright core but develops wide, energetic wings at large polar angles (Lazzati et al., 2017a; Lazzati et al., 2017b).

- Photospheric delay-After the outflow has broken out of the leading edge of the wind, it needs to propagate out to the photospheric radius. At this point the jet becomes transparent and the necessary conditions for the release of the prompt gamma-ray radiation are met. We indicate the delay due to the propagation from the break out radius to the photospheric radius as $\Delta t_{\mathrm{ph}}$.

- Dissipation delay-While at the photospheric radius the prompt emission can be radiated, it does not mean it is. In some models, such as the popular internal shock synchrotron model, the outflow needs to propagate out to the internal shock radius before the bulk energy of the flow is dissipated and turned into radiation. We indicate this additional delay as $\Delta t_{\gamma}$.

For the first time, a measurement of the sum of all these possible delays was available for GW170817 (Abbott et al., 2017b). The prompt gamma-ray radiation was detected with a delay $\Delta t_{\mathrm{GW}-\gamma} \simeq 1.75 \mathrm{~s}$. Several attempts have been made to constrain the various individual contributions, but a general consensus has not been achieved (Shoemaker and Murase, 2018; Gill et al., 2019b; Beniamini et al., 2020a; Hamidani et al., 2020). A few robust inferences can however be made (Lazzati et al., 2020). Overall, the measured delay was fairly small, since GW170817 ejected a significant amount of energy toward the observer but its Lorentz factor could be at most moderate $(\Gamma<7)$ (Beniamini et al., 2020b). These combine to a large photospheric radius and a photospheric delay

$$
\Delta t_{\mathrm{ph}} \sim \frac{R_{\mathrm{ph}}}{c \Gamma^{2}}=1.4 \frac{R_{\mathrm{ph}}}{2 \times 10^{12} \mathrm{~cm}}\left(\frac{7}{\Gamma}\right)^{2} \mathrm{~s}
$$

The photospheric delay therefore had to contribute to a sizable part of the delay, since any other non-photospheric emission mechanism would require a longer delay (this allows to use the above equation to put a Lower limit on $\Gamma$ (Beniamini 2020a). The wind delay, if there was any, had to be smaller than the jet delay, so that the jet-wind interaction could generate a structured outflow, as requested for modeling the afterglow emission. For the same reason, the jet delay itself could be fairly small but could not be null. Finally, the breakout and dissipation delays had to be small in order to accommodate the large expected photospheric delay. Note, however, that the prompt emission spectrum had a non-thermal shape, a property that is not expected form a simple photospheric emission model (see Section 3 for a more thorough discussion).

\subsection{The Shaping of the Outflow}

GW170817 was also the first GRB for which evidence of a structured outflow could be unequivocally determined. The structure of the outflow could be intrinsic, as the jet itself could have been launched with a non-uniform polar structure (Aloy et al., 2005; Kathirgamaraju et al., 2019). However, the relatively large energetics of GW170817 in gamma-rays and the shape of its afterglow lightcurve (see Section 4) suggest a wide structure, most likely brought about by the jet interaction with the wind from the merger (Lazzati and Perna, 2019; Salafia et al., 2020).

A typical SGRB jet with isotropic equivalent energy $E_{\text {iso }}=10^{53}$ erg and asymptotic Lorentz factor $\eta=100$ has a baryon rest mass $M_{0}=E_{\text {iso }} / \eta c^{2} \sim 10^{-4} M_{\odot}$. If it encounters a wind mass $M_{\text {wind }} \geq M_{0} / \eta \sim 10^{-6} M_{\odot}$ it is shocked and the velocity of propagation of its head is slowed until the working surface of the jet head is in causal contact, allowing for the wind material and the shocked jet material to move to the side instead of accumulating in front of the jet and thereby slowing it down (Matzner, 2003; Morsony et al., 2007; Bromberg et al., 2011; Lazzati and Perna, 2019). As a consequence, a high-pressure cocoon inflates around the jet, composed by partially mixed jet and wind material. As the jet breaks out of the wind leading edge, the cocoon loses the confining effect of the wind material and is released. Since it has large pressure, it accelerates creating a broad structure around the jet with decreasing energy and Lorentz factor for increasing polar angle. This process therefore turns a collimated jet into a structured outflow. It requires a small wind mass that is well below the expected amount of baryons ejected in a binary NS merger. The cocoon structure can be studied analytically, by enforcing pressure balance between the jet, cocoon, and wind material at their respective contact surfaces, or through numerical simulations. Despite its importance for 

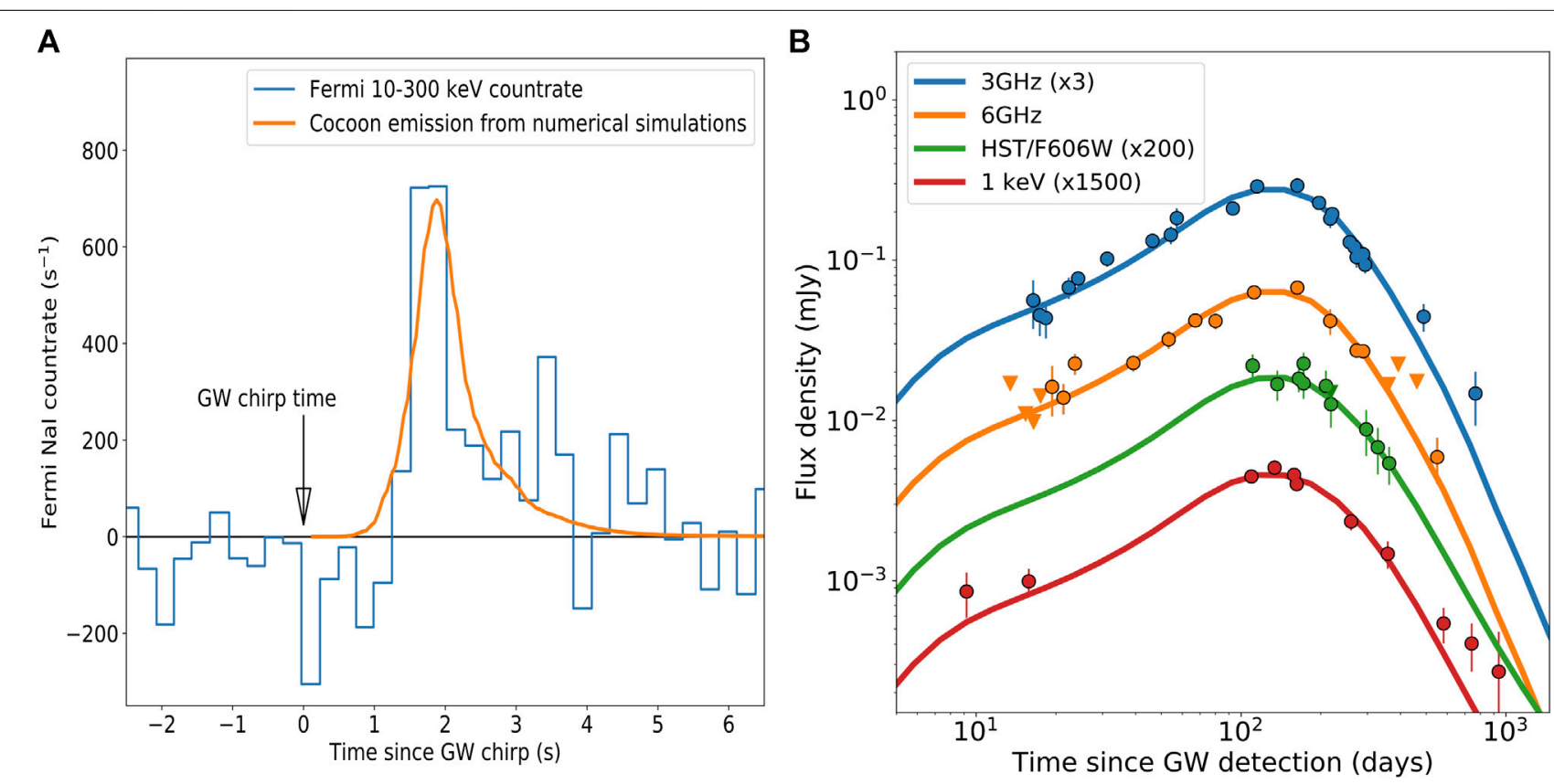

FIGURE 2 | Left panel (A): the prompt emission of GW170817. The blue step-line shows the Fermi data (Goldstein et al., 2017), while the orange solid line is the prediction from a theoretical simulation that assumes a structured outflow from the jet-wind interaction (Lazzati et al., 2017b). The radiation is assumed to be released at the photosphere. Right panel (B): Afterglow of GW170817. Symbols with error-bars show observations in the radio, optical, and X-ray bands. Solid lines show the best fit result for an afterglow model with a structured outflow and an observer located at $\theta_{0}=35^{\circ}$ from the line of sight. Additional data at different radio frequencies were used to constrain the model, but only two radio bands are shown for clarity. Adapted from Makhathini et al. (2020).

predicting burst/merger observability and understanding the structure and composition of the merger wind and jet, the polar profile of the outflow is highly debated. Analytic functions ranging from Gaussian, power-law, and exponential have been tested, and even numerical simulations do not provide an unequivocal answer (Murguia-Berthier et al., 2014; Nagakura et al., 2014; Lazzati et al., 2017b; Murguia-Berthier et al., 2017a; Murguia-Berthier et al., 2017b; Duffell et al., 2018; Granot et al., 2018a; Wu and MacFadyen, 2018; Xie et al., 2018; Geng et al., 2019; Gill et al., 2019a; Kathirgamaraju et al., 2019; Hamidani et al., 2020; Hamidani and Ioka, 2020; Murguia-Berthier et al., 2020; Takahashi and Ioka 2020a; Takahashi and Ioka 2020b).

\section{THE PROMPT EMISSION}

Approximately $1.75 \mathrm{~s}$ after the GW chirp, a gamma-ray pulse was observed by both the Fermi and INTEGRAL satellites from a position compatible with the direction from which the GWs arrived (Goldstein et al., 2017; Savchenko et al., 2017). The pulse was made by an initial spike of about half a second followed by a broader, less intense tail, for an overall duration of $\sim 2 \mathrm{~s}$. Two characteristics make this gamma-ray pulse different from the population of previously observed SGRBs: it is markedly less energetic than an average cosmological SGRB and, given its energetics, it has a very high peak frequency (Fong et al., 2015). As a matter of fact, the detection itself was surprising because the chance of having a SGRB jet pointing along the line of sight for the first GW-selected binary merger was expected to be small (Metzger and
Berger, 2012; Ghirlanda et al., 2016). That is because the amplitude of the GWs depend only mildly on the orientation of the binary, while the intensity of the radiation from a narrow, relativistic jet drops quickly for any line of sight outside the jet itself. Such an expectation was based, however, on the properties of a narrow jet and not on the possibility that the jet-wind interaction would cause a structured outflow to form. Predictions from models with structured outflows had indeed shown that, for moderately large off-axis angles, a detectable signal would be expected from a GWdetected merger (Lazzati et al., 2017a; Lazzati et al., 2017b). A similar effect might be responsible for X-ray flashes, when a long duration GRB is seen off-axis (Yamazaki 2020, Yamazaki 2003).

The structured outflow model was successful at predicting that a SGRB would be detectable even at large off-axis angles (Lazzati et al., 2017a; Lazzati et al., 2017b). It correctly predicted the offaxis burst energetics and its duration. It could also successfully explain the detected delay between the GWs and the $\gamma$-rays. A comparison between the Fermi data and the bolometric photospheric emission (Lazzati et al., 2017b) is shown in the left panel of Figure 2. The one aspect of GW170817 that cannot be accounted for by the simple photospheric cocoon emission is the $\gamma$-ray spectrum of the prompt emission. At least in first approximation, the photosphere of an off-axis structured outflow is expected to produce a thermal pulse with temperature (Lazzati et al., 2017a; Lazzati et al., 2017b)

$$
T_{\mathrm{obs}} \simeq\left(\frac{L \Gamma^{2}}{4 \pi \sigma R_{\mathrm{ph}}^{2}}\right)^{\frac{1}{4}}=10^{7}\left(\frac{L}{10^{47} \mathrm{erg}}\right)^{\frac{1}{4}}\left(\frac{\Gamma}{100}\right)^{\frac{1}{2}}\left(\frac{10^{12} \mathrm{~cm}}{\mathrm{R}_{\mathrm{ph}}}\right)^{\frac{1}{2}} \mathrm{~K}
$$


which would produce a spectrum peaked at a few $\mathrm{KeV}$, in severe tension with the observed peak frequency at $\sim 150 \mathrm{keV}$ (Goldstein et al., 2017). This is due to the fact that the cocoon, which energized the outflow at large off axis, is not expected to be radially structured, and therefore no significant dissipation is expected to occur around the photospheric radius, differently from the photospheres of long GRBs (Lazzati et al., 2009; Parsotan et al., 2018). One possible explanation is that the prompt radiation was due to an external shock (Veres et al., 2018). However, given the low Lorentz factor and low interstellar medium densities expected in the surroundings of GW170817, the timing of the prompt emission, less than $2 \mathrm{~s}$ after the launching of the jet, is difficult to explain. Alternatively, the prompt emission could be due to the breakout of the cocoon from the leading edge of the wind (Kasliwal et al., 2017; Bromberg et al., 2018; Gottlieb et al., 2018; Nakar et al., 2018). The shock breakout model can explain the energetics and spectrum of the prompt emission (Nakar and Sari, 2012) but requires a finely tuned setup in which the wind is very fast, so that it can reach a large enough radius at the breakout time. The origin of the prompt emission spectrum is therefore not been explained in a completely satisfactory way, yet (Kisaka et al., 2018; Meng et al., 2018; Pozanenko et al., 2018; Ioka and Nakamura, 2019; Matsumoto et al., 2019). The observation of more SGRBs from GWdetected mergers will offer further observational constraints to shed light on this remaining riddle.

\section{THE AFTERGLOW}

The afterglow of GW170817 had its own share of unique features. To begin with, it was not detected for more then a week, until it was bright enough to be seen first in X-rays (Margutti et al., 2017; Troja et al., 2017) and, at around the two weeks mark, in radio waves (Hallinan et al., 2017; Troja et al., 2017). The detection of the afterglow at optical wavelengths had to wait for the dimming of the associated $\mathrm{KN}$, and was performed only around day 110 with the Hubble Space Telescope (Lyman et al., 2018). Such late appearance of an afterglow is unprecedented, since the typical behavior is that the afterglow peaks very early, minutes to hours after the burst, and only dims with time afterward (van Paradijs et al., 2000; Nousek et al., 2006). A second unique feature of the afterglow of GW170817 was that, even after it was detected, it sustained a slow brightening at all wavelengths (Margutti et al., 2017; Mooley et al., 2018c; Ruan et al., 2018; Troja et al., 2019b), eventually peaking 150 days after the GW detection and dropping in luminosity steeply afterwords (Dobie et al., 2018; Makhathini et al., 2020) (see the right panel of Figure 2).

The outflow from GW170817 along the direction toward Earth was under-energetic by a factor 10,000 to 100,000 times with respect to a typical SGRB (Fong et al., 2017). An outstanding question was therefore whether GW170817 had a misaligned, SGRB-like jet pointing in a different direction or not (Lazzati et al., 2018; Mooley et al., 2018a; Salafia et al., 2018). If it did, then the identification of the SGRB progenitors with binary NS mergers would be secured. If did not, then what GW10817 was associated with would be a new class of dim, possibly isotropic, $\gamma$-ray transients. Unfortunately, telling whether a misaligned relativistic jet is present is not easy, since all the radiation is relativistically beamed away from the line of sight. The slow but steady brightening was shown to be consistent with the presence of a jet, its energy contribution along the line of sight growing with the deceleration of the external shock (Xiao et al., 2017; De Colle et al., 2018; Finstad et al., 2018; Granot et al., 2018b; Lamb and Kobayashi, 2018; Lazzati et al., 2018; Nakar et al., 2018; Fraija et al., 2019a; Fraija et al., 2019b; Beniamini et al., 2020b; Oganesyan et al., 2020). However, a radially stratified spherical outflow could reproduce the observations as well, albeit at the price of adding a never observed before component to the models (Li et al., 2018; Mooley et al., 2018c; Nakar et al., 2018; Nakar and Piran, 2018; Salafia et al., 2018). Some evidence in favor of a jet was provided by the steep post-peak decay at all wavelengths (Alexander et al., 2018; Jin et al., 2018; Lamb et al., 2018; Mooley et al., 2018b; Nynka et al., 2018; Fong et al., 2019; Hajela et al., 2019). In addition, it was soon realized that either a relatively large linear polarization (Gill and Granot, 2018) or a small but detectable proper motion of the radio transient could potentially give the final clue. Both observations were carried out. Polarization turned out to be small (Corsi et al., 2018), and only an upper limit of 12 per cent was obtained, still consistent with either explanation. Long baseline radio interferometry turned out to be the key. In one experiment, a small but significant proper motion was detected (Mooley et al., 2018a), while in a second experiment the radio source was confirmed to be point-like (Ghirlanda et al., 2019). Both these characteristics are incompatible with a spherical expansion. In the future, the detection of the counter-jet emission might give additional evidence (Yamazaki 2018).

To date, despite the very high quality of the available data, the unique afterglow of GW170817 can be modeled successfully with the good old external shock synchrotron model (Mészáros and Rees, 1997; Sari et al., 1998), with the only required addition of considering off-axis observers (Granot et al., 2002) and allowing for some structure in the polar direction (Lazzati et al., 2017b; Lazzati et al., 2018). The type of polar stratification is not univocally constrained, since Gaussian, power-law, and exponential profile seem all to give an adequate fit to the data (Lazzati et al., 2018; Troja et al., 2018a; Xie et al., 2018; Ghirlanda et al., 2019). Numerical simulations are also ambiguous, different codes yielding different polar structures, including the three mentioned above (Murguia-Berthier et al., 2014; Nagakura et al., 2014; Lazzati et al., 2017b; Murguia-Berthier et al., 2017a; MurguiaBerthier et al., 2017b; Duffell et al., 2018; Granot et al., 2018a; Wu and MacFadyen, 2018; Xie et al., 2018; Geng et al., 2019; Gill et al., 2019b; Kathirgamaraju et al., 2019; Hamidani et al., 2020). Constraints can be obtained from the lack of a large populations of cosmological off-axis bursts (Beniamini 2019). More observations and further theoretical work are needed to pin down this important aspect that has implications not only on the detectability of bursts but also on the nature of the inner engine and the composition of the ejected jet and wind. 


\section{SUMMARY, DISCUSSION, AND A LOOK AT THE FUTURE}

GW170817 was a rich event, a cornerstone detection in our understanding of SGRBs. It confirmed that binary NS mergers are the progenitor of at least some short bursts, it showed us that the top-hat jet model is woefully inadequate for describing the relativistic outflows of SGRBs (and possibly long duration GRBs as well) and it gave us, for the first time ever, a measure of the trigger time and of the delay between the launching of the jet and the detection of the prompt emission radiation.

We now know that the burst associated with GW170817 was a fairly canonical SGRB (Salafia et al., 2019), with a powerful relativistic jet that, after interacting with the merger wind, turned into a structured outflow (Lazzati et al., 2017b; Lazzati et al., 2018). Our line of sight lied somewhere between $15^{\circ}$ and $35^{\circ}$ away from the jet axis, the lower value obtained by high resolution radio imaging (Mooley et al., 2018c; Ghirlanda et al., 2019), while the larger value being favored by multi-band afterglow modeling and ejecta considerations (Lazzati et al., 2018; Mandel, 2018; Zou et al., 2018). The prompt emission was powered by an energetic cocoon inflated by the interaction of the jet with the merger wind. The gamma-ray radiation was likely released at or near the photosphere, either by a shock breakout (Nakar and Sari, 2012; Kasliwal et al., 2017; Gottlieb et al., 2018) or by other non-thermal mechanisms (Savchenko et al., 2017; Veres et al., 2018). The external shock developed later than usual due to the lower than customary Lorentz factor of the outflow along the line of sight and the afterglow was unusual, characterized by an initial increase in luminosity that lasted for a few months before peaking and beginning a steep declining phase. This behavior is understood to be due to the structure of the outflow, characterized by a polar stratification with a steep decline as a function of angle in both the energy per unit solid angle and the Lorentz factor.

Despite the large amount of observational evidence that allowed us to paint a detailed picture of the dynamic of the relativistic ejecta of GW170817 and their electromagnetic signatures, some questions remain open. First, we do not know the nature of the compact object that launched the relativistic jet. It could have been either a meta-stable NS or a $\mathrm{BH}$, and consensus in this respect hasn't been reached (Piro et al., 2019; Metzger et al., 2018; Pooley et al., 2018; Abedi and Afshordi, 2019). A related mystery is the origin of the observed

\section{REFERENCES}

Abbott, B. P., Abbott, R., Abbott, T. D., Acernese, F., Ackley, K., Adams, C., et al. (2017a). GW 170817: Observation of gravitational waves from a binary neutron star inspiral. Phys. Rev. Lett. 119, 161101. doi:10.1103/PhysRevLett. 119.161101

Abbott, B. P., Abbott, R., Abbott, T. D., Acernese, F., Ackley, K., Adams, C., et al. (2017b). Gravitational waves and gamma-rays from a binary neutron star merger: GW170817 and GRB 170817A. Astrophys. J. 848, L13. doi:10.3847/ 2041-8213/aa920c

Abbott, B. P., Abbott, R., Abbott, T. D., Acernese, F., Ackley, K., Adams, C., et al. $(2017 \mathrm{c})$. Multi-messenger observations of a binary neutron star merger. Astrophys. J. 848, L12. doi:10.3847/2041-8213/aa91c9
$1.75 \mathrm{~s}$ delay between the GW and the prompt emission. As discussed in Section $\mathbf{2 . 1}$ the delay is the sum of many components and it is unclear which dominates, or if several of them have comparable magnitude. Since the photospheric delay is strongly dependent on the viewing angle, observation of several SGRBs from a diverse set of angles will help better understand the origin of the delay. Still unclear is also the physics of the dissipation that powered the prompt emission and the prompt emission mechanism itself. Shock breakouts, internal dissipation such as internal shocks, and even external shocks have been proposed (see Section 3).

Finally, we still do not know how typical GW170817 was. The fact that most likely it originated from a binary NS merger does not exclude the possibility that some-if not most-SGRB are made in NS-BH mergers. It might even be that GW170817 itself was a NS-BH merger (Coughlin and Dietrich, 2019; Kyutoku et al., 2020). Re-analysis of several past bursts have yielded some support the presence of kilonovae in their light curves (Troja et al., 2018b; Beniamini et al., 2019; Lamb et al., 2019b; Troja et al., 2019a) or similarities in their prompt emission (Burns 2018, von Kienlin 2019), showing that GW170817 was not unique. However, there might be cases in which the jet is not successful in breaking out of the wind leading edge, and a weaker transient would be produced (Kasliwal et al., 2017; Mooley et al., 2018c; Salafia et al., 2018). Future GW detections with the power of multimessenger observations will allow to better understand the connection between binary NS mergers, binary NS-BH mergers, and SGRBs.

\section{AUTHOR CONTRIBUTIONS}

DL has written this review article on his own to the best of his knowledge, he has produced all figures himself from publicly available data and codes. The references are extensive but they are by no means exhaustive.

\section{FUNDING}

DL acknowledges support from NASA grants 80NSSC18K1729 (Fermi) and NNX17AK42G (ATP), Chandra grant TM9-20002X, and NSF grant AST-1907955.

Abdalla, H., Abramowski, A., Aharonian, F., Ait Benkhali, F., Angüner, E. O., Arakawa, M., et al. (2017). TeV gamma-ray observations of the binary neutron star merger GW170817 with H.E.S.S. Astrophys. J. Lett. 850, L22. doi:10.3847/ 2041-8213/aa97d2

Abedi, J., andAfshordi, N. (2019). Echoes from the abyss: a highly spinning black hole remnant for the binary neutron star merger GW170817. J. Cosmol. Astropart. Phys. 2019, 010. doi:10.1088/1475-7516/2019/11/010

Alexander, K. D., Berger, E., Fong, W., Williams, P. K. G., Guidorzi, C., Margutti, R., et al. (2017). The electromagnetic counterpart of the binary neutron star merger LIGO/virgo GW170817. VI. Radio constraints on a relativistic jet and predictions for late-time emission from the kilonova ejecta. Astrophys. J. 848, L21. doi:10.3847/2041-8213/aa905d

Alexander, K. D., Margutti, R., Blanchard, P. K., Fong, W., Berger, E., Hajela, A., et al. (2018). A decline in the X-ray through radio emission from GW170817 
continues to support an off-axis structured jet. Astrophys. J. 863, L18. doi:10. 3847/2041-8213/aad637

Aloy, M. A., Janka, H.-T., andMüller, E. (2005). Relativistic outflows from remnants of compact object mergers and their viability for short gamma-ray bursts. Astron. Astrophys. 436, 273-311. doi:10.1051/0004-6361:20041865

Arcavi, I., Hosseinzadeh, G., Howell, D. A., McCully, C., Poznanski, D., Kasen, D., et al. (2017). Optical emission from a kilonova following a gravitational-wavedetected neutron-star merger. Nature 551, 64-66. doi:10.1038/nature24291

Barkov, M. V., andPozanenko, A. S. (2011). Model of the extended emission of short gamma-ray bursts. Mon. Not. R. Astron. Soc. 417, 2161-2165. doi:10. 1111/j.1365-2966.2011.19398.x

Belczynski, K., Perna, R., Bulik, T., Kalogera, V., Ivanova, N., andLamb, D. Q. (2006). A study of compact object mergers as short gamma-ray burst progenitors. Astrophys. J. 648, 1110-1116. doi:10.1086/505169

Beniamini, P., Duran, R. B., Petropoulou, M., andGiannios, D. (2020a). Ready, set, launch: time interval between a binary neutron star merger and short gamma-ray burst jet formation. Astrophys. J. 895, L33. doi:10.3847/2041$8213 /$ ab 9223

Beniamini, P., Granot, J., andGill, R. (2020b). Afterglow light curves from misaligned structured jets. Mon. Not. R. Astron. Soc. 493, 3521-3534. doi:10. 1093/mnras/staa538

Beniamini, P., andNakar, E. (2019). Observational constraints on the structure of gamma-ray burst jets. mnras 482 (4), 5430-5440. doi:10.1093/mnras/sty3110.

Beniamini, P., Petropoulou, M., Barniol Duran, R., andGiannios, D. (2019). A lesson from GW170817: most neutron star mergers result in tightly collimated successful GRB jets. Mon. Not. R. Astron. Soc. 483, 840-851. doi:10.1093/ mnras/sty3093

Bromberg, O., Nakar, E., Piran, T., andSari, R. E. (2011). The propagation of relativistic jets in external media. Astrophys. J. 740, 100. doi:10.1088/0004$637 \mathrm{X} / 740 / 2 / 100$

Bromberg, O., Nakar, E., Piran, T., andSari, R. E. (2013). Short versus long and collapsars versus non-collapsars: a quantitative classification of gamma-ray bursts. Astrophys. J. 764, 179. doi:10.1088/0004-637X/764/2/179

Bromberg, O., Tchekhovskoy, A., Gottlieb, O., Nakar, E., andPiran, T. (2018). The $\gamma$-rays that accompanied GW170817 and the observational signature of a magnetic jet breaking out of NS merger ejecta. Mon. Not. R. Astron. Soc. 475, 2971-2977. doi:10.1093/mnras/stx3316

Bucciantini, N., Quataert, E., Arons, J., Metzger, B. D., andThompson, T. A. (2008). Relativistic jets and long-duration gamma-ray bursts from the birth of magnetars. Mon. Not. R. Astron. Soc. Lett. 383, L25-L29. doi:10.1111/j.17453933.2007.00403.x

Bucciantini, N., Quataert, E., Metzger, B. D., Thompson, T. A., Arons, J., andDel Zanna, L. (2009). Magnetized relativistic jets and long-duration GRBs from magnetar spin-down during core-collapse supernovae. Mon. Not. R. Astron. Soc. 396, 2038-2050. doi:10.1111/j.1365-2966.2009.14940.x

Burns, E., Veres, P., Connaughton, V., Racusin, J., Briggs, M.-S., and Christensen, N. (2018). Fermi GBM observations of GRB 150101B: a second nearby event with a short hard spike and a soft tail. apjl 863 (2), L34. doi:10.3847/2041-8213/ aad813.

Ciolfi, R. (2018). Short gamma-ray burst central engines. Int. J. Mod. Phys. 27, 1842004. doi:10.1142/S021827181842004X

Ciolfi, R., andSiegel, D. M. (2015). Short gamma-ray bursts in the "time-reversal" scenario. Astrophys. J. 798, L36. doi:10.1088/2041-8205/798/2/L36

Cook, G. B., Shapiro, S. L., andTeukolsky, S. A. (1994). Rapidly rotating polytropes in general relativity. Astrophys. J. 422, 227-242. doi:10.1086/173721

Corsi, A., Hallinan, G. W., Lazzati, D., Mooley, K. P., Murphy, E. J., Frail, D. A., et al. (2018). An upper limit on the linear polarization fraction of the GW170817 radio continuum. Astrophys. J. 861, L10. doi:10.3847/2041-8213/ aacdfd

Coughlin, M. W., andDietrich, T. (2019). Can a black hole-neutron star merger explain GW170817, AT2017gfo, and GRB170817A? Phys. Rev. D 100, 043011. doi:10.1103/PhysRevD.100.043011

Coulter, D. A., Foley, R. J., Kilpatrick, C. D., Drout, M. R., Piro, A. L., Shappee, B. J., et al. (2017). Swope Supernova Survey 2017a (SSS17a), the optical counterpart to a gravitational wave source. Science $358,1556-1558$. doi:10.1126/science. aap9811

Cowperthwaite, P. S., Berger, E., Villar, V. A., Metzger, B. D., Nicholl, M., Chornock, R., et al. (2017). The electromagnetic counterpart of the binary neutron star merger LIGO/virgo GW170817. II. UV, optical, and near-infrared light curves and comparison to kilonova models. Astrophys. J. 848, L17. doi:10. 3847/2041-8213/aa8fc7

Dainotti, M. G., Hernandez, X., Postnikov, S., Nagataki, S., O’brien, P., Willingale, R., et al. (2017). A study of the gamma-ray burst fundamental plane. Astrophys. J. 848, 88. doi:10.3847/1538-4357/aa8a6b

Dainotti, M. G., Willingale, R., Capozziello, S., Cardone, V. F., andOstrowski, M . (2010). Discovery of a tight correlation for gamma-ray burst afterglows with "canonical" light curves. Astrophys. J. 722, L215-L219. doi:10.1088/2041-8205/ $722 / 2 / \mathrm{L} 215$

D’Avanzo, P., Campana, S., Salafia, O. S., Ghirland, A. G., Ghisellini, G., Melandri, A., et al. (2018). The evolution of the X-ray afterglow emission of GW 170817/ GRB 170817A in XMM-Newton observations. Astron. Astrophys. 613, L1. doi:10.1051/0004-6361/201832664

De Colle, F., Kumar, P., andAguilera-Dena, D. R. (2018). Radio emission from the cocoon of a GRB jet: implications for relativistic supernovae and off-axis GRB emission. Astrophys. J. 863, 1. doi:10.3847/1538-4357/aad04d

Dobie, D., Kaplan, D. L., Murphy, T., Lenc, E., Mooley, K. P., Lynch, C., et al. (2018). A turnover in the radio light curve of GW170817. Astrophys. J. 858, L15. doi:10.3847/2041-8213/aac105

Duffell, P. C., Quataert, E., Kasen, D., andKlion, H. (2018). Jet dynamics in compact object mergers: GW170817 likely had a successful jet. Astrophys. J. 866, 1. doi:10.3847/1538-4357/aae084

Evans, P. A., Cenko, S. B., Kennea, J. A., Emery, S. W. K., Kuin, N. P. M., Korobkin, O., et al. (2017). SwiftandNuSTARobservations of GW170817: detection of a blue kilonova. Science 358, 1565-1570. doi:10.1126/science.aap9580

Finstad, D., De, S., Brown, D. A., Berger, E., andBiwer, C. M. (2018). Measuring the viewing angle of GW170817 with electromagnetic and gravitational waves. Astrophys. J. 860, L2. doi:10.3847/2041-8213/aac6c1

Fishman, G. J., andMeegan, C. A. (1995). Gamma-ray bursts. Annu. Rev. Astron. Astrophys. 33, 415-458. doi:10.1146/annurev.aa.33.090195.002215

Fong, W., Blanchard, P. K., Alexander, K. D., Strader, J., Margutti, R., Hajela, A., et al. (2019). The optical afterglow of GW170817: an off-axis structured jet and deep constraints on a globular cluster origin. Astrophys. J. 883, L1. doi:10.3847/ 2041-8213/ab3d9e

Fong, W., Berger, E., Blanchard, P. K., Margutti, R., Cowperthwaite, P. S., Chornock, R., et al. (2017). The electromagnetic counterpart of the binary neutron star merger LIGO/virgo GW170817. VIII. A comparison to cosmological short-duration gamma-ray bursts. Astrophys. J. 848, L23. doi:10.3847/2041-8213/aa9018

Fong, W., andBerger, E. (2013). The locations of short gamma-ray bursts as evidence for compact object binary progenitors. Astrophys. J. 776, 18. doi:10. 1088/0004-637X/776/1/18

Fong, W., Berger, E., Margutti, R., andZauderer, B. A. (2015). A decade of shortduration gamma-ray burst broadband Afterglows: energetics, circumburst densities, and jet opening angles. Astrophys. J. 815, 102. doi:10.1088/0004$637 \mathrm{X} / 815 / 2 / 102$

Fraija, N., Colle, F. D., Veres, P., Dichiara, S., Duran, R. B., Galvan-Gamez, A., et al. (2019a). The short GRB 170817A: modeling the off-axis emission and implications on the ejecta magnetization. Astrophys. J. 871, 123. doi:10.3847/ 1538-4357/aaf564

Fraija, N., Pedreira, A. C. C. D. E. S., andVeres, P. (2019b). Light curves of a shockbreakout material and a relativistic off-axis jet from a binary neutron star system. Astrophys. J. 871, 200. doi:10.3847/1538-4357/aaf80e

Gehrels, N., Ramirez-Ruiz, E., andFox, D. B. (2009). Gamma-ray bursts in theSwiftEra. Annu. Rev. Astron. Astrophys. 47, 567-617. doi:10.1146/ annurev.astro.46.060407.145147

Geng, J. J., Zhang, B., Kölligan, A., Kuiper, R., andHuang, Y. F. (2019). Propagation of a short GRB jet in the ejecta: jet launching delay time, jet structure, and GW170817/GRB 170817A. Astrophys. J. 877, L40. doi:10.3847/2041-8213/ab224b

Ghirlanda, G., Salafia, O. S., Paragi, Z., Giroletti, M., Yang, J., Marcote, B., et al. (2019). Compact radio emission indicates a structured jet was produced by a binary neutron star merger. Science 363, 968-971. doi:10.1126/science. aau8815

Ghirlanda, G., Salafia, O. S., Pescalli, A., Ghisellini, G., Salvaterra, R., Chassande-Mottin, E., et al. (2016). Short gamma-ray bursts at the dawn of the gravitational wave era. Astron. Astrophys. 594, A84. doi:10.1051/0004-6361/ 201628993 
Giacomazzo, B., Perna, R., Rezzolla, L., Troja, E., andLazzati, D. (2013). Compact binary progenitors of short gamma-ray bursts. Astrophys. J. 762, L18. doi:10. $1088 / 2041-8205 / 762 / 2 / \mathrm{L} 18$

Gill, R., andGranot, J. (2018). Afterglow imaging and polarization of misaligned structured GRB jets and cocoons: breaking the degeneracy in GRB 170817A. Mon. Not. R. Astron. Soc. 478, 4128-4141. doi:10.1093/mnras/sty1214

Gill, R., Granot, J., De Colle, F., andUrrutia, G. (2019a). Numerical simulations of an initially top-hat jet and the afterglow of GW170817/GRB170817A. Astrophys. J. 883, 15. doi:10.3847/1538-4357/ab3577

Gill, R., Nathanail, A., andRezzolla, L. (2019b). When did the remnant of GW170817 collapse to a black hole? Astrophys. J. 876, 139. doi:10.3847/ $1538-4357 / \mathrm{ab} 16 \mathrm{da}$

Goldstein, A., Veres, P., Burns, E., Briggs, M. S., Hamburg, R., Kocevski, D., et al. (2017). An ordinary short gamma-ray burst with extraordinary implications: Fermi -GBM detection of GRB 170817A. Astrophys. J. 848, L14. doi:10.3847/ 2041-8213/aa8f41

Gottlieb, O., Nakar, E., Piran, T., andHotokezaka, K. (2018). A cocoon shock breakout as the origin of the $\gamma$-ray emission in GW170817. Mon. Not. R. Astron. Soc. 479, 588-600. doi:10.1093/mnras/sty1462

Granot, J., De Colle, F., andRamirez-Ruiz, E. (2018a). Off-axis afterglow light curves and images from 2D hydrodynamic simulations of double-sided GRB jets in a stratified external medium. Mon. Not. R. Astron. Soc. 481, 2711-2720. doi:10.1093/mnras/sty2454

Granot, J., Gill, R., Guetta, D., andDe Colle, F. (2018b). Off-axis emission of short GRB jets from double neutron star mergers and GRB 170817A. Mon. Not. R. Astron. Soc. 481, 1597-1608. doi:10.1093/mnras/sty2308

Granot, J., Guetta, D., andGill, R. (2017). Lessons from the short GRB 170817A: the first gravitational-wave detection of a binary neutron star merger. Astrophys. J. 850, L24. doi:10.3847/2041-8213/aa991d

Granot, J., Panaitescu, A., Kumar, P., andWoosley, S. E. (2002). Off-axis afterglow emission from jetted gamma-ray bursts. Astrophys. J. 570, L61-L64. doi:10. $1086 / 340991$

Haggard, D., Nynka, M., Ruan, J. J., Kalogera, V., Cenko, S. B., Evans, P., et al. (2017). A deep Chandra X-ray study of neutron star coalescence GW170817. Astrophys. J. 848, L25. doi:10.3847/2041-8213/aa8ede

Hajela, A., Margutti, R., Alexander, K. D., Kathirgamaraju, A., Baldeschi, A., Guidorzi, C., et al. (2019). Two years of nonthermal emission from the binary neutron star merger GW170817: rapid fading of the jet afterglow and first constraints on the kilonova fastest ejecta. Astrophys. J. 886, L17. doi:10.3847/ 2041-8213/ab5226

Hallinan, G., Corsi, A., Mooley, K. P., Hotokezaka, K., Nakar, E., Kasliwal, M. M., et al. (2017). A radio counterpart to a neutron star merger. Science 358, 1579-1583. doi:10.1126/science.aap9855

Hamidani, H., andIoka, K. (2020). Jet propagation in expanding medium for gamma-ray bursts. arXiv e-prints.

Hamidani, H., Kiuchi, K., andIoka, K. (2020). Jet propagation in neutron star mergers and GW170817. Mon. Not. R. Astron. Soc. 491, 3192-3216. doi:10. 1093/mnras/stz3231

Ioka, K., andNakamura, T. (2019). Spectral puzzle of the off-axis gamma-ray burst in GW170817. Mon. Not. R. Astron. Soc. 487, 4884-4889. doi:10.1093/mnras/ stz1650

Jin, Z.-P., Li, X., Wang, H., Wang, Y.-Z., He, H.-N., Yuan, Q., et al. (2018). Short GRBs: opening angles, local neutron star merger rate, and off-axis events for GRB/GW association. Astrophys. J. 857, 128. doi:10.3847/1538-4357/aab76d

Kasen, D., Metzger, B., Barnes, J., Quataert, E., andRamirez-Ruiz, E. (2017). Origin of the heavy elements in binary neutron-star mergers from a gravitational-wave event. Nature 551, 80-84. doi:10.1038/nature24453

Kasliwal, M. M., Nakar, E., Singer, L. P., Kaplan, D. L., Cook, D. O., Van Sistine, A., et al. (2017). Illuminating gravitational waves: a concordant picture of photons from a neutron star merger. Science 358, 1559-1565. doi:10.1126/science.aap9455

Kathirgamaraju, A., Tchekhovskoy, A., Giannios, D., andBarniol Duran, R. (2019). EM counterparts of structured jets from 3D GRMHD simulations. Mon. Not. R. Astron. Soc. 484, L98-L103. doi:10.1093/mnrasl/slz012

Kim, S., Schulze, S., Resmi, L., González-López, J., Higgins, A. B., Ishwara-Chandra, C. H., et al. (2017). ALMA and GMRT constraints on the off-axis gamma-ray burst 170817A from the binary neutron star merger GW170817. Astrophys. J. 850, L21. doi:10.3847/2041-8213/aa970b
Kisaka, S., Ioka, K., Kashiyama, K., andNakamura, T. (2018). Scattered short gamma-ray bursts as electromagnetic counterparts to gravitational waves and implications of GW170817 and GRB 170817A. Astrophys. J. 867, 39. doi:10. 3847/1538-4357/aae30a

Kiuchi, K., Sekiguchi, Y., Shibata, M., andTaniguchi, K. (2010). Exploring binary-neutron-star-merger scenario of short-gamma-ray bursts by gravitational-wave observation. Phys. Rev. Lett. 104, 141101. doi:10. 1103/PhysRevLett.104.141101

Kouveliotou, C., Meegan, C. A., Fishman, G. J., Bhat, N. P., Briggs, M. S., Koshut, T. M., et al. (1993). Identification of two classes of gamma-ray bursts. Astrophys. J. 413, L101. doi:10.1086/186969

Kumar, P., andZhang, B. (2015). The physics of gamma-ray bursts and relativistic jets. Phys. Rep. 561, 1-109. doi:10.1016/j.physrep.2014.09.008

Kyutoku, K., Fujibayashi, S., Hayashi, K., Kawaguchi, K., Kiuchi, K., Shibata, M., et al. (2020). On the possibility of GW190425 being a black hole-neutron star binary merger. Astrophys. J. 890, L4. doi:10.3847/2041-8213/ab6e70

Lamb, G. P., andKobayashi, S. (2018). GRB 170817A as a jet counterpart to gravitational wave triggerGW 170817. Mon. Not. R. Astron. Soc. 478, 733-740. doi:10.1093/mnras/sty1108

Lamb, G. P., Lyman, J. D., Levan, A. J., Tanvir, N. R., Kangas, T., Fruchter, A. S., et al. (2019a). The optical afterglow of GW170817 at one year post-merger. Astrophys. J. 870, L15. doi:10.3847/2041-8213/aaf96b

Lamb, G. P., Mandel, I., andResmi, L. (2018). Late-time evolution of afterglows from off-axis neutron star mergers. Mon. Not. R. Astron. Soc. 481, 2581-2589. doi:10.1093/mnras/sty2196

Lamb, G. P., Tanvir, N. R., Levan, A. J., Postigo, A. D. U., Kawaguchi, K., Corsi, A., et al. (2019b). Short GRB 160821B: a reverse shock, a refreshed shock, and a well-sampled kilonova. Astrophys. J. 883, 48. doi:10.3847/1538-4357/ ab38bb

Lasota, J.-P., Haensel, P., andAbramowicz, M. A. (1996). Fast rotation of neutron stars. Astrophys. J. 456, 300. doi:10.1086/176650

Lazzati, D., Ciolfi, R., andPerna, R. (2020). Intrinsic properties of the engine and jet that powered the short gamma-ray burst associated with GW170817. Astrophys. J. 898, 1-59. doi:10.3847/1538-4357/ab9a44

Lazzati, D., Deich, A., Morsony, B. J., andWorkman, J. C. (2017a). Off-axis emission of short $\gamma$-ray bursts and the detectability of electromagnetic counterparts of gravitational-wave-detected binary mergers. Mon. Not. R. Astron. Soc. 471, 1652-1661. doi:10.1093/mnras/stx1683

Lazzati, D., López-Cámara, D., Cantiello, M., Morsony, B. J., Perna, R., andWorkman, J. C. (2017b). Off-axis prompt X-ray transients from the cocoon of short gamma-ray bursts. Astrophys. J. 848, L6. doi:10.3847/2041$8213 / \mathrm{aa} 8 \mathrm{f} 3 \mathrm{~d}$

Lazzati, D., Morsony, B. J., andBegelman, M. C. (2009). Very high efficiency photospheric emission in long-duration $\gamma$-ray bursts. Astrophys. J. 700, L47-L50. doi:10.1088/0004-637X/700/1/L47

Lazzati, D., andPerna, R. (2019). Jet-cocoon outflows from neutron star mergers: structure, light curves, and fundamental physics. Astrophys. J. 881, 89. doi:10. 3847/1538-4357/ab2e06

Lazzati, D., Perna, R., Morsony, B. J., Lopez-Camara, D., Cantiello, M., Ciolfi, R., et al. (2018). Late time afterglow observations reveal a collimated relativistic jet in the ejecta of the binary neutron star merger GW170817. Phys. Rev. Lett. 120, 241103. doi:10.1103/PhysRevLett.120.241103

Lee, H. K., Wijers, R. A. M. J., andBrown, G. E. (2000). The Blandford-Znajek process as a central engine for a gamma-ray burst. Phys. Rep. 325, 83-114. doi:10.1016/S0370-1573(99)00084-8

Lee, W. H., andRamirz-Ruiz, E. (2007). The progenitors of short gamma-ray bursts. New J. Phys. 9, 17. doi:10.1088/1367-2630/9/1/017

Lei, W.-H., Zhang, B., andLiang, E.-W. (2013). Hyperaccreting black hole as gamma-ray burst central engine. I. Baryon loading in gamma-ray burst jets. Astrophys. J. 765, 125. doi:10.1088/0004-637X/765/2/125

Li, B., Li, L.-B., Huang, Y.-F., Geng, J.-J., Yu, Y.-B., andSong, L.-M. (2018). Continued brightening of the afterglow of GW170817/GRB 170817A as being due to a delayed energy injection. Astrophys. J. 859, L3. doi:10.3847/ 2041-8213/aac2c5

Lin, D.-B., Liu, T., Lin, J., Wang, X.-G., Gu, W.-M., andLiang, E.-W. (2018). First electromagnetic pulse associated with a gravitational-wave event: profile, duration, and delay. Astrophys. J. 856, 90. doi:10.3847/1538-4357/aab3d7 
Liu, T., Romero, G. E., Liu, M.-L., andLi, A. (2016). Fast radio bursts and their gamma-ray or radio afterglows as kerr-newman black hole binaries. . apj 826 (1), 82.. doi:10.3847/0004-637X/826/1/82.

Lü, H.-J., Liang, E.-W., Zhang, B.-B., andZhang, B. (2010). A new classification method for gamma-ray bursts. Astrophys. J. 725, 1965-1970. doi:10.1088/0004637X/725/2/1965

Lucca, M., and Sagunski, L. (2020). The lifetime of binary neutron star merger remnants. J. High Energy Astrophys. 27, 33-37. doi:10.1016/j.jheap.2020.04.003.

Lyman, J. D., Lamb, G. P., Levan, A. J., Mandel, I., Tanvir, N. R., Kobayashi, S., et al. (2018). The optical afterglow of the short gamma-ray burst associated with GW170817. Nat. Astron. 2, 751-754. doi:10.1038/s41550-018-0511-3

Makhathini, S., Mooley, K. P., Brightman, M., Hotokezaka, K., Nayana, A., Intema, H. T., et al. (2020). The Panchromatic Afterglow of GW170817: the full uniform dataset, modeling, comparison with previous results and implications. arXiv e-prints arXiv:2006.02382.

Mandel, I. (2018). The orbit of GW170817 was inclined by less than 280 to the line of sight. Astrophys. J. 853, L12. doi:10.3847/2041-8213/aaa6c1

Margutti, R., Alexander, K. D., Xie, X., Sironi, L., Metzger, B. D., Kathirgamaraju, A., et al. (2018). The binary neutron star event LIGO/virgo GW170817 160 Days after merger: synchrotron emission across the electromagnetic spectrum. Astrophys. J. 856, L18. doi:10.3847/2041-8213/aab2ad

Margutti, R., Berger, E., Fong, W., Guidorzi, C., Alexander, K. D., Metzger, B. D., et al. (2017). The electromagnetic counterpart of the binary neutron star merger LIGO/virgo GW170817. V. Rising X-ray emission from an off-axis jet. Astrophys. J. 848, L20. doi:10.3847/2041-8213/aa9057

Matsumoto, T., Nakar, E., andPiran, T. (2019). Constraints on the emitting region of the gamma-rays observed in GW170817. Mon. Not. R. Astron. Soc. 483, 1247-1255. doi:10.1093/mnras/sty3200

Matzner, C. D. (2003). Supernova hosts for gamma-ray burst jets: dynamical constraints. Mon. Not. R. Astron. Soc. 345, 575-589. doi:10.1046/j.1365-8711. 2003.06969.x

Meegan, C., Lichti, G., Bhat, P. N., Bissaldi, E., Briggs, M. S., Connaughton, V., et al. (2009). Thefermigamma-ray burst monitor. Astrophys. J. 702, 791-804. doi:10. 1088/0004-637X/702/1/791

Meng, Y.-Z., Geng, J.-J., Zhang, B.-B., Wei, J.-J., Xiao, D., Liu, L.-D., et al. (2018). The origin of the prompt emission for short GRB 170817A: photosphere emission or synchrotron emission? Astrophys. J. 860, 72. doi:10.3847/1538-4357/aac2d9

Mészáros, P. (2002). Theories of gamma-ray bursts. Annu. Rev. Astron. Astrophys. 40, 137-169. doi:10.1146/annurev.astro.40.060401.093821

Mészáros, P., andRees, M. J. (1997). Optical and long-wavelength afterglow from gamma-ray bursts. Astrophys. J. 476, 232-237. doi:10.1086/303625

Metzger, B. D., andBerger, E. (2012). What is the most promising electromagnetic counterpart of a neutron star binary merger? Astrophys. J. 746, 48. doi:10.1088/ 0004-637X/746/1/48

Metzger, B. D., Giannios, D., Thompson, T. A., Bucciantini, N., andQuataert, E. (2011). The protomagnetar model for gamma-ray bursts. Mon. Not. R. Astron. Soc. 413, 2031-2056. doi:10.1111/j.1365-2966.2011.18280.x

Metzger, B. D., Thompson, T. A., andQuataert, E. (2018). A magnetar origin for the kilonova ejecta in GW170817. Astrophys. J. 856, 101. doi:10.3847/1538-4357/ aab095

Mooley, K. P., Deller, A. T., Gottlieb, O., Nakar, E., Hallinan, G., Bourke, S., et al. (2018a). Superluminal motion of a relativistic jet in the neutron-star merger GW170817. Nature 561, 355-359. doi:10.1038/s41586-018-0486-3

Mooley, K. P., Frail, D. A., Dobie, D., Lenc, E., Corsi, A., De, K., et al. (2018b). A strong jet signature in the late-time light curve of GW170817. Astrophys. J. 868, L11. doi:10.3847/2041-8213/aaeda7

Mooley, K. P., Nakar, E., Hotokezaka, K., Hallinan, G., Corsi, A., Frail, D. A., et al. (2018c). A mildly relativistic wide-angle outflow in the neutron-star merger event GW170817. Nature 554, 207-210. doi:10.1038/nature25452

Morsony, B. J., Lazzati, D., andBegelman, M. C. (2007). Temporal and angular properties of gamma-ray burst jets emerging from massive stars. Astrophys. J. 665, 569-598. doi:10.1086/519483

Murguia-Berthier, A., Montes, G., Ramirez-Ruiz, E., De Colle, F., andLee, W. H. (2014). Necessary conditions for short gamma-ray burst production in binary neutron star mergers. Astrophys. J. 788, L8. doi:10.1088/2041-8205/788/1/L8

Murguia-Berthier, A., Ramirez-Ruiz, E., De Colle, F., Janiuk, A., Rosswog, S., andLee, W. H. (2020). The fate of the merger remnant in GW170817 and its imprint on the jet structure. arXiv e-prints.
Murguia-Berthier, A., Ramirez-Ruiz, E., Kilpatrick, C. D., Foley, R. J., Kasen, D., Lee, W. H., et al. (2017a). A neutron star binary merger model for GW170817/GRB 170817A/SSS17a. Astrophys. J. 848, L34. doi:10.3847/ 2041-8213/aa91b3

Murguia-Berthier, A., Ramirez-Ruiz, E., Montes, G., De Colle, F., Rezzolla, L., Rosswog, S., et al. (2017b). The properties of short gamma-ray burst jets triggered by neutron star mergers. Astrophys. J. 835, L34. doi:10.3847/2041$8213 /$ aa5b9e

Nagakura, H., Hotokezaka, K., Sekiguchi, Y., Shibata, M., andIoka, K. (2014). Jet collimation in the ejecta of double neutron star mergers: a new canonical picture of short gamma-ray bursts. Astrophys. J. 784, L28. doi:10.1088/2041$8205 / 784 / 2 / \mathrm{L} 28$

Nakar, E., Gal-Yam, A., andFox, D. B. (2006). The local rate and the progenitor lifetimes of short-hard gamma-ray bursts: synthesis and predictions for the laser interferometer gravitational-wave observatory. Astrophys. J. 650, 281-290. doi:10.1086/505855

Nakar, E., Gottlieb, O., Piran, T., Kasliwal, M. M., andHallinan, G. (2018). From $\gamma$ to radio: the electromagnetic counterpart of GW170817. Astrophys. J. 867, 18. doi:10.3847/1538-4357/aae205

Nakar, E., andPiran, T. (2018). Implications of the radio and X-ray emission that followed GW170817. Mon. Not. R. Astron. Soc. 478, 407-415. doi:10.1093/ mnras/sty952

Nakar, E., andSari, R. E. (2012). Relativistic shock breakouts-a variety of gammaray flares: from low-luminosity gamma-ray bursts to type ia supernovae. Astrophys. J. 747, 88. doi:10.1088/0004-637X/747/2/88

Norris, J. P., andBonnell, J. T. (2006). Short gamma-ray bursts with extended emission. Astrophys. J. 643, 266-275. doi:10.1086/502796

Norris, J. P., Gehrels, N., andScargle, J. D. (2010). Threshold for extended emission in short gamma-ray bursts. Astrophys. J. 717, 411-419. doi:10.1088/0004-637X/ $717 / 1 / 411$

Nousek, J. A., Kouveliotou, C., Grupe, D., Page, K. L., Granot, J., Ramirez-Ruiz, E., et al. (2006). Evidence for a canonical gamma-ray burst afterglow light curve in theSwiftXRT data. Astrophys. J. 642, 389-400. doi:10.1086/500724

Nynka, M., Ruan, J. J., Haggard, D., andEvans, P. A. (2018). Fading of the X-ray afterglow of neutron star merger GW170817/GRB 170817A at 260 days. Astrophys. J. 862, L19. doi:10.3847/2041-8213/aad32d

Oganesyan, G., Ascenzi, S., Branchesi, M., Salafia, O. S., Dall'Osso, S., andGhirlanda, G. (2020). Structured jets and X-ray plateaus in gamma-ray burst phenomena. Astrophys. J. 893, 88. doi:10.3847/1538-4357/ab8221

Parsotan, T., López-Cámara, D., andLazzati, D. (2018). Photospheric emission from variable engine gamma-ray burst simulations. Astrophys. J. 869, 103. doi:10.3847/1538-4357/aaeed1

Perna, R., Lazzati, D., andGiacomazzo, B. (2016). Short gamma-ray bursts from the merger of two black holes. Astrophys. J. 821, L18. doi:10.3847/2041-8205/821/1/L18

Pian, E., D’Avanzo, P., Benetti, S., Branchesi, M., Brocato, E., Campana, S., et al. (2017). Spectroscopic identification of r-process nucleosynthesis in a double neutron-star merger. Nature 551, 67-70. doi:10.1038/nature24298

Piran, T. (1999). Gamma-ray bursts and the fireball model. Phys. Rep. 314, 575-667. doi:10.1016/S0370-1573(98)00127-6

Piro, L., Troja, E., Zhang, B., Ryan, G., van Eerten, H., Ricci, R., et al. (2019). A longlived neutron star merger remnant in GW170817: constraints and clues from X-ray observations. Mon. Not. R. Astron. Soc. 483, 1912-1921. doi:10.1093/ mnras/sty3047

Pooley, D., Kumar, P., Wheeler, J. C., andGrossan, B. (2018). GW170817 most likely made a black hole. Astrophys. J. 859, L23. doi:10.3847/2041-8213/aac3d6

Pozanenko, A. S., Barkov, M. V., Minaev, P. Y., Volnova, A. A., Mazaeva, E. D., Moskvitin, A. S., et al. (2018). GRB 170817A associated with GW170817: multifrequency observations and modeling of prompt gamma-ray emission. Astrophys. J. 852, L30. doi:10.3847/2041-8213/aaa2f6

Ramirez-Ruiz, E., Celotti, A., andRees, M. J. (2002). Events in the life of a cocoon surrounding a light, collapsar jet. Mon. Not. R. Astron. Soc. 337, 1349-1356. doi:10.1046/j.1365-8711.2002.05995.x

Resmi, L., Schulze, S., Ishwara-Chandra, C. H., Misra, K., Buchner, J., Pasquale, M. D., et al. (2018). Low-frequency view of GW170817/GRB 170817A with the giant metrewave radio telescope. Astrophys. J. 867, 57. doi:10.3847/1538-4357/aaela6

Ruan, J. J., Nynka, M., Haggard, D., Kalogera, V., andEvans, P. (2018). Brightening X-ray emission from GW170817/GRB 170817A: further evidence for an outflow. Astrophys. J. 853, L4. doi:10.3847/2041-8213/aaa4f3 
Ruiz, M., Lang, R. N., Paschalidis, V., andShapiro, S. L. (2016). Binary neutron star mergers: a jet engine for short gamma-ray bursts. Astrophys. J. 824, L6. doi:10. 3847/2041-8205/824/1/L6

Salafia, O. S., Barbieri, C., Ascenzi, S., andToffano, M. (2020). Gamma-ray burst jet propagation, development of angular structure, and the luminosity function. Astron. Astrophys. 636, A105. doi:10.1051/0004-6361/201936335

Salafia, O. S., Ghirlanda, G., Ascenzi, S., andGhisellini, G. (2019). On-axis view of GRB 170817A. Astron. Astrophys. 628, A18. doi:10.1051/0004-6361/201935831

Salafia, O. S., Ghisellini, G., Ghirlanda, G., andColpi, M. (2018). Interpreting GRB170817A as a giant flare from a jet-less double neutron star merger. Astron. Astrophys. 619, A18. doi:10.1051/0004-6361/201732259

Sari, R. E., Piran, T., andNarayan, R. (1998). Spectra and light curves of gamma-ray burst afterglows. Astrophys. J. 497, L17-L20. doi:10.1086/311269

Savchenko, V., Ferrigno, C., Kuulkers, E., Bazzano, A., Bozzo, E., Brandt, S., et al. (2017). INTEGRAL detection of the first prompt gamma-ray signal coincident with the gravitational-wave event GW170817. Astrophys. J. 848, L15. doi:10. 3847/2041-8213/aa8f94

Schutz, B. F. (2011). Networks of gravitational wave detectors and three figures of merit. Class. Quantum Gravity. 28, 125023. doi:10.1088/0264-9381/28/12/125023

Shoemaker, I. M., andMurase, K. (2018). Constraints from the time lag between gravitational waves and gamma rays: implications of GW170817 and GRB 170817A. Phys. Rev. D 97, 083013. doi:10.1103/PhysRevD.97.083013

Siebert, M. R., Foley, R. J., Drout, M. R., Kilpatrick, C. D., Shappee, B. J., Coulter, D. A., et al. (2017). The unprecedented properties of the first electromagnetic counterpart to a gravitational-wave source. Astrophys. J. 848, L26. doi:10.3847/ 2041-8213/aa905e

Smartt, S. J., Chen, T. W., Jerkstrand, A., Coughlin, M., Kankare, E., Sim, S. A., et al. (2017). A kilonova as the electromagnetic counterpart to a gravitational-wave source. Nature 551, 75-79. doi:10.1038/nature24303

Soares-Santos, M., Holz, D. E., Annis, J., Chornock, R., Herner, K., Berger, E., et al. (2017). The electromagnetic counterpart of the binary neutron star merger LIGO/virgo GW170817. I. Discovery of the optical counterpart using the dark energy camera. Astrophys. J. 848, L16. doi:10.3847/2041-8213/aa9059

Tanvir, N. R., Levan, A. J., González-Fernández, C., Korobkin, O., Mandel, I., Rosswog, S., et al. (2017). The emergence of a lanthanide-rich kilonova following the merger of two neutron stars. Astrophys. J. 848, L27. doi:10. 3847/2041-8213/aa90b6

Takahashi, K., andIoka, K. (2020a). Diverse jet structures consistent with the offaxis afterglow of GRB 170817A. arXiv e-prints.

Takahashi, K., andIoka, K. (2020b). Inverse reconstruction of jet structure from offaxis gamma-ray burst afterglows. mnras. 497 (1), 1217-1235. doi:10.1093/ mnras/staa1984.

Troja, E., Castro-Tirado, A. J., González, J. B., Hu, Y., Ryan, G. S., Cenko, S. B., et al. (2019a). The afterglow and kilonova of the short GRB 160821B. Mon. Not. R. Astron. Soc. 489, 2104-2116. doi:10.1093/mnras/stz2255

Troja, E., Piro, L., Ryan, G., van Eerten, H., Ricci, R., Wieringa, M. H., et al. (2018a). The outflow structure of GW170817 from late-time broad-band observations. Mon. Not. R. Astron. Soc. 478, L18-L23. doi:10.1093/mnrasl/sly061

Troja, E., Piro, L., van Eerten, H., Wollaeger, R. T., Im, M., Fox, O. D., et al. (2017). The X-ray counterpart to the gravitational-wave event GW170817. Nature 551, 71-74. doi:10.1038/nature24290

Troja, E., Ryan, G., Piro, L., van Eerten, H., Cenko, S. B., Yoon, Y., et al. (2018b). A luminous blue kilonova and an off-axis jet from a compact binary merger at $\mathrm{z}=$ 0.1341. Nat. Commun. 9, 4089. doi:10.1038/s41467-018-06558-7

Troja, E., van Eerten, H., Ryan, G., Ricci, R., Burgess, J. M., Wieringa, M. H., et al. (2019b). A year in the life of GW170817: the rise and fall of a structured jet from a binary neutron star merger. Mon. Not. R. Astron. Soc. 489, 1919-1926. doi:10. 1093/mnras/stz2248

Valenti, S., Sand, D. J., Yang, S., Cappellaro, E., Tartaglia, L., Corsi, A., et al. (2017). The discovery of the electromagnetic counterpart of GW170817: kilonova at 2017gfo/DLT17ck. Astrophys. J. 848, L24. doi:10.3847/2041-8213/aa8edf

van Paradijs, J., Kouveliotou, C., andWijers, R. A. M. J. (2000). Gamma-ray burst afterglows. Annu. Rev. Astron. Astrophys. 38, 379-425. doi:10.1146/annurev. astro.38.1.379
Veres, P., Mészáros, P., Goldstein, A., Fraija, N., Connaughton, V., Burns, E., et al. (2018). Gamma-ray burst models in light of the GRB 170817A - GW170817 connection. arXiv e-prints arXiv:1802.07328.

Vietri, M., andStella, L. (1998). A gamma-ray burst model with small baryon contamination. Astrophys. J. 507, L45-L48. doi:10.1086/311674

Villar, V. A., Cowperthwaite, P. S., Berger, E., Blanchard, P. K., Gomez, S., Alexander, K. D., et al. (2018). Spitzer Space telescope infrared observations of the binary neutron star merger GW170817. Astrophys. J. 862, L11. doi:10. 3847/2041-8213/aad281

von Kienlin, A., Veres, P., Roberts, O.-J., Hamburg, R., Bissaldi, E. et al. (2019). Fermi-GBM GRBs with Characteristics Similar to GRB 170817A. apj 876 (1), 89. doi:10.3847/1538-4357/ab10d8.

Woosley, S. E. (1993). Gamma-ray bursts from stellar mass accretion disks around black holes. Astrophys. J. 405, 273. doi:10.1086/172359

Woosley, S. E., andBloom, J. S. (2006). The supernova-gamma-ray burst connection. Annu. Rev. Astron. Astrophys. 44, 507-556. doi:10.1146/ annurev.astro.43.072103.150558

Woosley, S. E., andHeger, A. (2006). The progenitor stars of gamma-ray bursts. Astrophys. J. 637, 914-921. doi:10.1086/498500

$\mathrm{Wu}, \mathrm{Y}$., andMacFadyen, A. (2018). Constraining the outflow structure of the binary neutron star merger event GW170817/GRB170817A with a Markov chain Monte Carlo analysis. Astrophys. J. 869, 55. doi:10.3847/1538-4357/ aae9de

Xiao, D., Liu, L.-D., Dai, Z.-G., andWu, X.-F. (2017). Afterglows and kilonovae associated with nearby low-luminosity short-duration gamma-ray bursts: application to GW170817/GRB 170817A. Astrophys. J. 850, L41. doi:10. 3847/2041-8213/aa9b2b

Xie, X., Zrake, J., andMacFadyen, A. (2018). Numerical simulations of the jet dynamics and synchrotron radiation of binary neutron star merger event GW170817/GRB 170817A. Astrophys. J. 863, 58. doi:10.3847/1538-4357/ aacf9c

Yamazaki, R., Ioka, K., andNakamura, T. (2018). Prompt emission from the counter jet of a short gamma-ray burst. Prog. Theor. Exp. Phys. 2018 (3), 033E01 doi:10.1093/ptep/pty012.

Yamazaki, R., Ioka, K., andNakamura, T. (2020). X-ray flashes from off-axis gamma-ray bursts. apjl 571 (1), L31-L35. doi:10.1086/341225.

Yamazaki, R., Yonetoku, D., andNakamura, T. (2003). An off-axis jet model for GRB 980425 and low-energy gamma-ray bursts. apjl 594 (2), L79-L82. doi:10.1086/ 378736.

Yoon, S.-C., Langer, N., andNorman, C. (2006). Single star progenitors of long gamma-ray bursts. Astron. Astrophys. 460, 199-208. doi:10.1051/0004-6361: 20065912

Zhang, B. (2016). Mergers of charged black holes: gravitational-wave events, short gamma-ray bursts, and fast radio bursts. Astrophys. J. 827, L31. doi:10.3847/ 2041-8205/827/2/L31

Zhang, B. (2019). The delay time of gravitational wave-gamma-ray burst associations. Front. Phys. 14, 64402. doi:10.1007/s11467-019-0913-4

Zhang, B.-B., Zhang, B., Sun, H., Lei, W.-H., Gao, H., Li, Y., et al. (2018). A peculiar low-luminosity short gamma-ray burst from a double neutron star merger progenitor. Nat. Commun. 9, 447. doi:10.1038/s41467-018-02847-3

Zou, Y.-C., Wang, F.-F., Moharana, R., Liao, B., Chen, W., Wu, Q., et al. (2018). Determining the Lorentz factor and viewing angle of GRB 170817A. Astrophys. J. 852 L1. doi:10.3847/2041-8213/aaa123

Conflict of Interest: The authors declare that the research was conducted in the absence of any commercial or financial relationships that could be construed as a potential conflict of interest.

Copyright (C) 2020 Lazzati. This is an open-access article distributed under the terms of the Creative Creative Commons Attribution License (CC BY). The use, distribution or reproduction in other forums is permitted, provided the original author(s) and the copyright owner(s) are credited and that the original publication in this journal is cited, in accordance with accepted academic practice. No use, distribution or reproduction is permitted which does not comply with these terms. 